\title{
Management Platform for Next Generation Optical Networks
}

\author{
Krzysztof Janicki, Pawel Mrozicki, Pawel Wiatr \\ Comarch S.A., Al. Jana Pawła II 39a, 31-864 Kraków, Poland \\ \{krzysztof.janicki, pawel.mrozicki, pawel.wiatr\}@comarch.com
}

\begin{abstract}
This paper presents Comarch's work performed in the frame of Eureka/Celtic project MANGO - Management platform for Next Generation Optical networks, where we define and create a management platform for all-optical networks. The main issues considered within the project are (a) cross-layer automated fault and performance management, (b) complex Service Quality management and (c) management of optical connections with appropriate support from the management system. In this paper we describe how the optical services can be defined and monitored within the platform. We describe the data model including physical impairments to be used by Routing and Wavelength Assignment (RWA) algorithms performing optical path computation.
\end{abstract}

Keywords: management of optical networks, fault management, alarm correlation, optical services modelling optical services management.

\section{INTRODUCTION}

The functionality of currently available all-optical networks is limited due to several reasons; one of them is the lack of an appropriate management platform. Management Software platforms are developed mainly by the companies producing optical network equipment. The software is designed only for managing their specific optical networks elements. Our approach is to consider the network management from the service management perspective that is not limited to the particular manufacturer. Consequently, the new management platform will be an integrated platform combining several functionalities of the management system. We present our work and results in defining and creating such a platform within the MANGO project. The platform is intended to provide support for handling information that is necessary for the proper operation of optical networks. The main issues tackled within the project are: (a) cross-layer automated fault and performance management, (b) complex services monitoring and (c) management of optical connections. In further paragraphs all these issues are presented in more detail.

MANGO project consortium consists of project partners from universities (The Royal Institute of Technology KTH, Stockholm, Sweden and Warsaw University of Technology, WUT, Poland), research laboratory (Acreo, Stockholm, Sweden), telecommunication provider (TeliaSonera, Sweden), and private companies (Proximion, Sweden and, Comarch, Poland).

\section{System architecture}

The proposed integrated management platform comprises both hardware and software components delivered by different partners of the project. Comarch OSS Suite, currently used to manage non-optical communication networks, is one of them. Comarch OSS Suite is the major software component of the MANGO platform. It is being extended to cover functionalities of optical network management and integrated with software components from other partners. The MANGO platform is schematically shown in the Fig. 1.

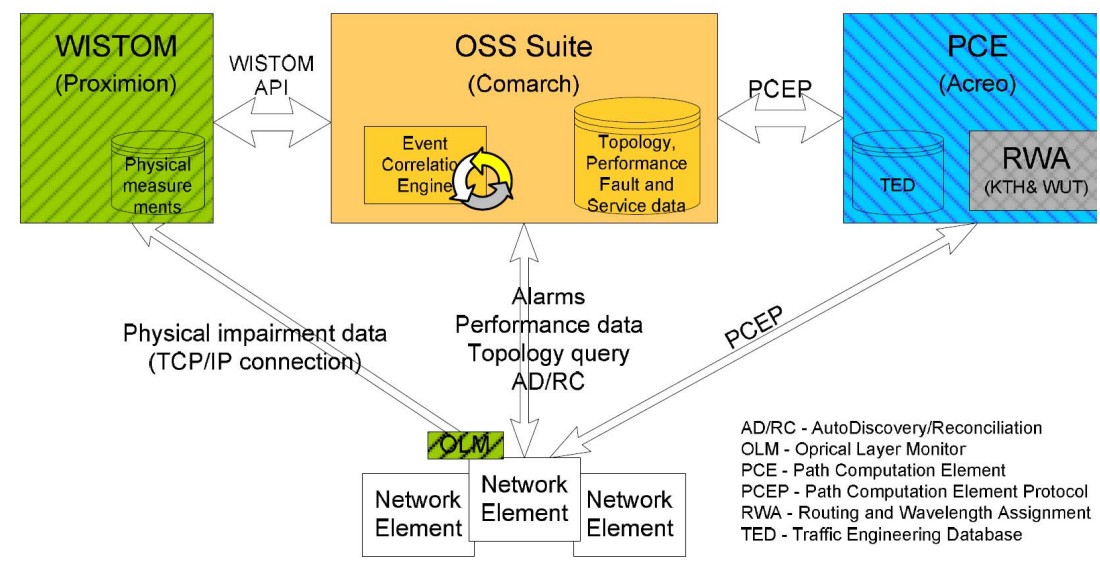

Fig. 1. System architecture.

WISTOM optical performance monitoring platform provided by Proximion supplies Comarch OSS Suite with necessary physical signal quality measures (e.g. impairments data) that can be used for Service Monitoring and Path Computation. In the extended version, Comarch OSS Suite keeps track on optical network equipment (Network Elements), connections topology (cables, fibres and lightpaths), alarms, and performance data. The 
data in Comarch OSS Suite is represented in a hierarchical, object-based model where data types are specified as objects. There are abstract objects with associated sets of parameters and relations to detailed objects (for example a switch or router are subtypes of physical device). Within the project the data model has been extended to provide functionality required for the project in particular to handle the following objects: Optical fibre Distribution Frame (ODF), Amplifiers (EDFA and SOA), Optical multiplexers and de-multiplexers (including ROADM), Optical Transponders, Optical Cross-Connects (OXC), Multi-Degree ROADM, and Dispersion Compensating Module (DCM).

In addition, the fibres have been categorised and modelled in Comarch OSS Suite based on their type described in ITU-T G.65x [1] series of recommendations. Layers representing optical network have been added to provide appropriate visualization.

A model which covers the optical transport network system consists of both physical elements (manholes, ductworks, pipes, optical cables with fibres and physical devices) and logical objects (termination points, trails in different layers). Below a simple example is presented: an SDH trail set between locations A and $\mathrm{C}$ through location $\mathrm{B}$ and its decomposition into fibre connections.

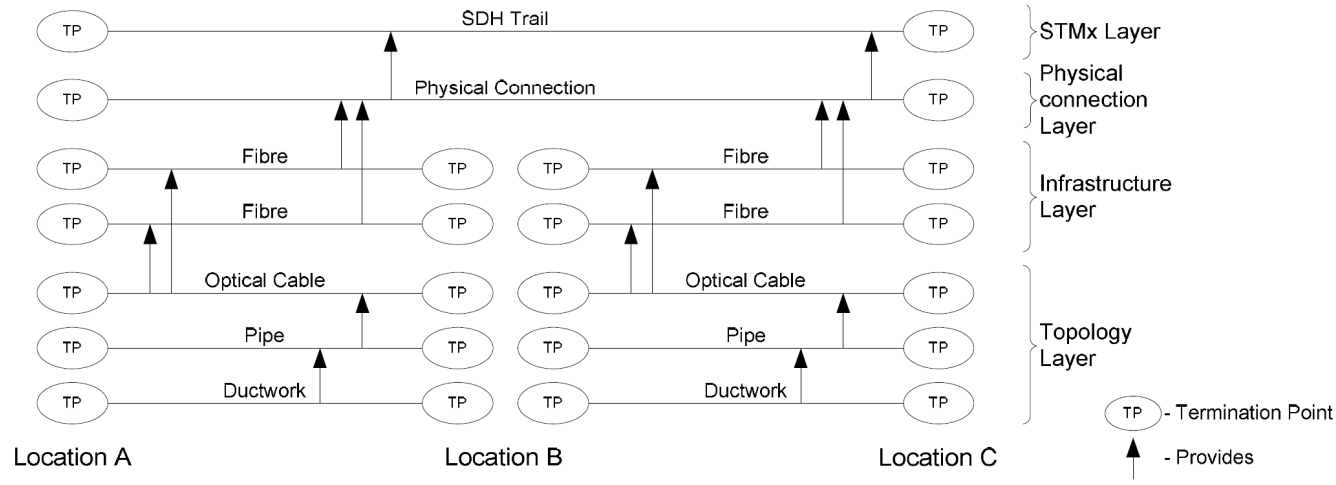

Fig. 2. Layered approach in MANGO platform.

Simple model for the WDM connection is presented in the Fig. 3 below. The physical connection layer is decomposed into infrastructure. The physical connection is provided by DWDM link and nonmultiplexed signals(lambdas) injects in particular fibres.

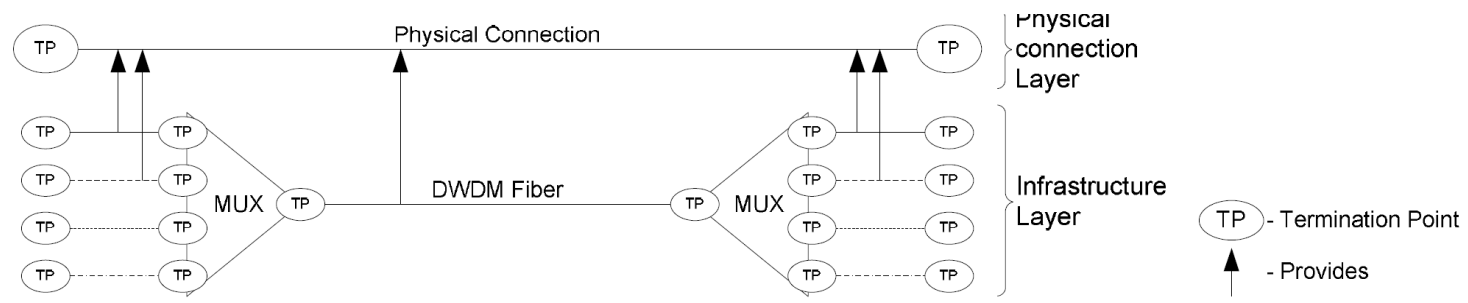

Fig. 3. DWDM Modelling.

Comarch OSS Suite allows Service Modeling and Monitoring basing on a predefined service model that includes alarm and Key Performance Indicator propagation rules.

Finally, the last components of the MANGO platform, the Path Computation Element $[2,3,4]$ part and in particular the RWA algorithms are studied by Acreo, KTH and WUT.

\section{Cross-layer automated fault and performance management}

Next generation optical networks are able to transport hundreds of high bandwidth wavelength channels (lightpaths) through a single optical fiber. As a result, a fiber failure may cause an interruption of a huge number of connections and therefore, a loss of tremendous amount of data. Therefore, fast fault detection and diagnosis followed by recovery mechanisms is crucial. For this purpose we have proposed the Comarch Correlation Engine that has been successfully verified in the environment of traditional operators. The Engine allows easy definition and implementation of cross-layer fault correlations in optical networks, together with exemplary correlations and actions. The system provides the Known Event Database (KEDB) where the user can gather and extend correlations, and define actions executed on particular event or correlation.

From the fault management point of view the most important issue was to analyze the types of alarms that are characteristic for optical networks and the principles that should govern the correlation of those alarms and the root-cause analysis of errors in the optical networks. We have analyzed the types of alarms generated by the optical network equipment, in particular the alarms generated by equipment that will be used in the pilot phase of the project, i.e. the equipment manufactured by the Siemens (HiT7300) series and Transcom (TM series). 
The alarms have been classified into five categories depending on the type of alarm:

- equipment alarms pertaining directly to optical hardware elements (cards, shelves, etc.);

- communication alarms pertaining to termination points of transport entities and functions; processing error alarms pertaining to network elements (NE) software;

- environmental alarms pertaining to telemetry sensors that monitor conditions/events external to the NE;

- QoS alarms pertaining to termination points at which performance measurement and threshold supervision is enabled.

In total more than 120 different types of alarms have been recognized; the potential severity of those alarms, potential trouble-shooting procedures and impact on the higher layers has also been examined.

The major issues pertaining to the area of performance and service management are physical layer impairments measurements. The following impairment types have been considered: polarization mode dispersion (PMD), chromatic dispersion (CD), amplifier spontaneous emission noise (ASE) and switching node crosstalk (SC). Some of these parameters are currently monitored by the WISTOM module and are being integrated with Comarch OSS Suite for service parameters monitoring.

\section{Service management}

Monitoring of the performance parameters in optical networks differs from monitoring in "traditional" telecommunication networks. In all-optical transparent networks services given by the optical layer, e.g. optical circuits (lightpaths), require monitoring of a different set of parameters. The parameters that need to be taken into account include optical signal quality indicators (e.g. power level, noise, polarization mode dispersion (PMD), etc.). Although the Service Quality parameters are different, the service monitoring can be based on a similar idea as in the traditional networks. Therefore, we utilize the existing Service Monitoring platform in Comarch OSS Suite and extend it to handle optical services. The services can be modeled in the system in a hierarchy decomposing the service into internal services and resources. The modeling of the services is covered by the Comarch OSS Suite data model. The classical concepts of Customer Facing Service - CFS - representing the service offered to the client and Resource Facing Service - RFC - representing real network components that are used to provide higher level service, are followed. Within the service model the propagation rules for events (alarms) and performance data can be defined.

The example of Alien wavelength service configured within Comarch OSS Suite together with alarm and performance propagation rules is presented in the Fig. 4

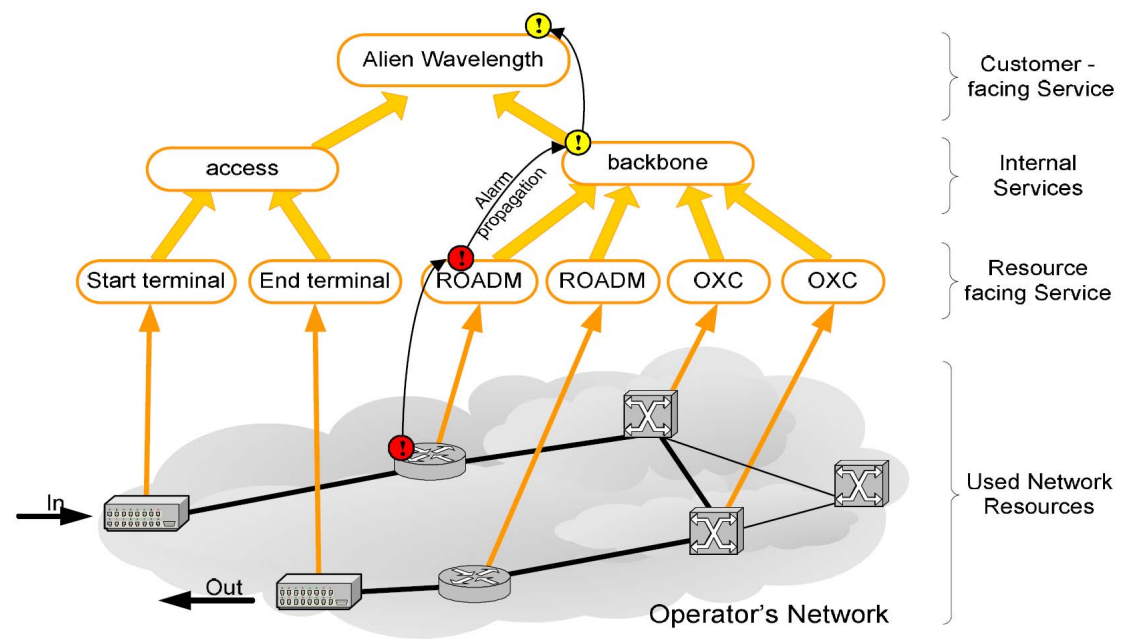

Fig. 4. High level model of alien wavelength service with propagation rules.

The set of services realized by the optical network encompasses two classes of services: services realized by the optical layer itself, and services realized by the higher layers. The following services of the optical layer have been analyzed:

- dark fiber service,

- alien (optically transparent) wavelength service,

- framed (not optically transparent) wavelength service with specified digital client format(s).

The analysis has resulted in defining the SLA parameters of those services. Moreover, the problems of managing entire SLA lifecycle for every service instance been analyzed. Service lifecycle consists of the following phases: service development, negotiation and sales, SLA implementation, SLA and service monitoring and SLA assessment. 


\section{Optical connections management}

The absence of electronic signal regenerators in all-optical networks imposes the need for taking into account the optical signal impairment constraint during the Routing and Wavelength Assignment (RWA) phase. Due to the lack of electrical regeneration, and the evolution towards a scenario where optical signals are routed, added/dropped, multiplexed, and de-multiplexed in the optical layer, optical signal impairments may accumulate along a lightpath. In this context the configuration management functionalities will provide connectionprovisioning tools able to account for physical impairments when performing the RWA.

For each type of the considered impairment the mathematical model of the signal deterioration phenomena has been analyzed. It has also been examined if the total value of the impairment encountered on the optical connection can be expressed in a simplest function of the impairment values encountered on the individual links of the connection; this problem is crucial from the point of view of the RWA algorithms.

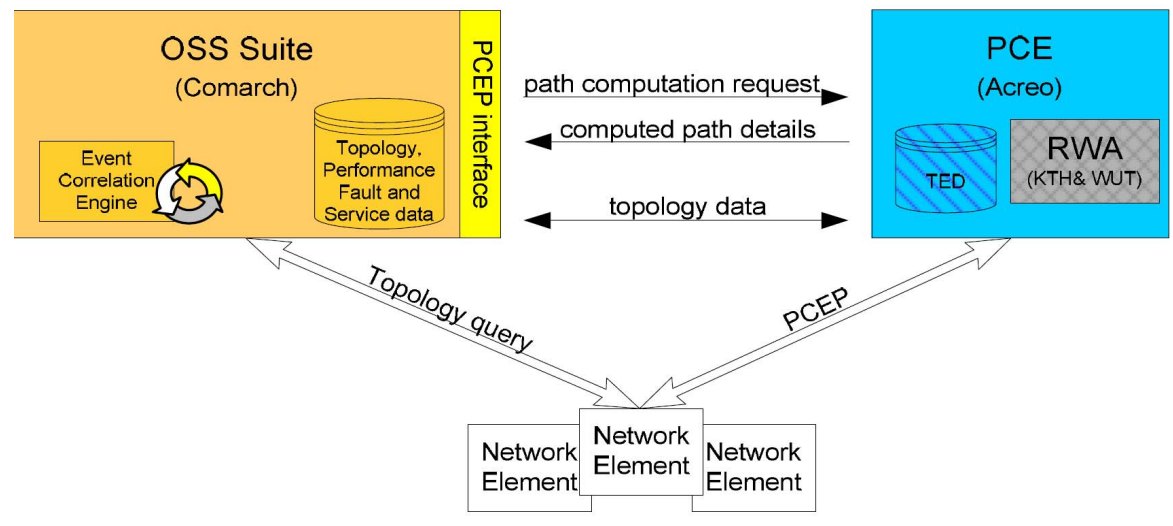

Fig. 5. Management of optical connections.

In the Comarch OSS a proper modeling of optical connections has been added. Moreover, adequate attributes for impairments have been created. Appropriate interfaces for the PCE protocol have been analyzed and are currently being developed. The management system communicates with the Path Computation Module (PCM) by these interfaces. Connections created by the PCM are stored in the inventory module of the NMS. The idea for management of optical connection is presented in the Fig. 5.

\section{Conclusions and future works}

In the frame of the MANGO project Comarch has extended the commercial Comarch OSS Suite in several ways. The application can handle optical connections (fibers and cables) with impairments information, optical equipments and specific optical networks alarms.

Future works in the MANGO project will involve the implementation of a fully integrated Path Computation Module (PCM) which, by proper interfaces, will use information from Comarch OSS Suite and will be able to automatically compute and set the proper lightpaths connections in optical networks.

The experience gathered during the project will provide an input for further developments of the management platform that, after the completion of this project, will be extended and packaged to fulfill the requirements that are requested by the market.

\section{ACKNOWLEDGEMENTS}

We would like to acknowledge our partners Acreo, KTH, Proximion, TeliaSonera and WUT for very fruitful cooperation within the MANGO project. In particular, we would like to thank Lena Wosinska from KTH, Sweden for encouragement in preparing this article, suggestions and fruitful discussions.

\section{REFERENCES}

[1] [1]ITU-T Recommendation G.65x series: Transmission systems and media, digital systems and networks. Transmission media characteristics - Optical fiber cables 1997-2000.

[2] [2]A. Farrel, J. Vasseur, and J. Ash, "A path computation element (PCE)-based architecture", RFC 4655, August 2006.

[3] [3] Y. Lee, G. Bernstein, and D. Li, "Alternative approaches to traffic engineering database creation and maintenance for path computation elements", draft-lee-pce-ted-alternatives-01 (work in progress), February 2009.

[4] [4]J. Vasseur and J. Le Roux, "Path computation element (PCE) communication protocol (PCEP)", RFC 5440, March 2009. 